\title{
Psychological profile and unexpected pain in oral lichen planus: A case-control multicenter SIPMO study ${ }^{a}$
}

\author{
Daniela Adamo $^{1}$ (D) | Elena Calabria ${ }^{1}$ (D) | Noemi Coppola ${ }^{1}$ | Lorenzo Lo Muzio $^{2}$ | \\ Michele Giuliani $^{2}$ | Maria Eleonora Bizzoca ${ }^{2}$ | Lorenzo Azzi ${ }^{3}$ (D) | Fabio Croveri ${ }^{3}$ | \\ Giuseppe Colella $^{4}$ | Ciro Emiliano Boschetti ${ }^{4}$ | Lucio Montebugnoli ${ }^{5}$ | Davide Gissi ${ }^{5}$ | \\ Mario Gabriele $^{6}$ | Marco Nisi ${ }^{6}$ | Andrea Sardella ${ }^{7}$ (D) | Giovanni Lodi ${ }^{7}$ (D) | \\ Elena M. Varoni ${ }^{7}$ | Amerigo Giudice ${ }^{8}$ (D) | Alessandro Antonelli ${ }^{8}$ | Marco Cabras ${ }^{9}$ (D) | \\ Alessio Gambino $^{9}$ | Paolo Vescovi ${ }^{10}$ | Alessandra Majorana ${ }^{11}$ | Elena Bardellini ${ }^{11}$ \\ Giuseppina Campisi $^{12}$ | Vera Panzarella ${ }^{12}$ | Spadari Francesco ${ }^{13}$ | Sonia Marino ${ }^{13}$ | \\ Monica Pentenero ${ }^{14}$ (i) | Marco Ardore ${ }^{14}$ | Matteo Biasotto ${ }^{15}$ | Margherita Gobbo ${ }^{15,16}$ \\ Luca Guarda Nardini $^{16}$ | Umberto Romeo ${ }^{17}$ | Gianluca Tenore ${ }^{17}$ | Rosario Serpico ${ }^{4}$ | \\ Carlo Lajolo $^{18}$ | Gioele Gioco ${ }^{18}$ (D) | Massimo Aria ${ }^{19}$ | Michele Davide Mignogna ${ }^{1}$ | \\ SIPMO (Italian Society of Oral Pathology, Medicine) ${ }^{1}$
}

\footnotetext{
${ }^{1}$ Department of Neurosciences, Reproductive and Odontostomatological Sciences, University of Naples Federico II, Naples, Italy

${ }^{2}$ Department of Clinical and Experimental Medicine, University of Foggia, Foggia, Italy

${ }^{3}$ Unit of Oral Medicine and Pathology, ASST dei Sette Laghi, Department of Medicine and Surgery, University of Insubria, Varese, Italy

${ }^{4}$ Multidisciplinary Department of Medical, Surgical and Dental Specialties, University of Campania Luigi Vanvitelli, Naples, Italy

${ }^{5}$ Department of Biomedical and Neuromotor Sciences, Section of Oral Sciences, University of Bologna, Bologna, Italy

${ }^{6}$ Department of Surgical Pathology, Medicine, Molecular and Critical Area, University of Pisa, Pisa, Italy

${ }^{7}$ Department of Biomedical, Surgical and Dental Sciences, University of Milan, Milan, Italy

${ }^{8}$ Department of Health Sciences, Magna Graecia University of Catanzaro, Catanzaro, Italy

${ }^{9}$ Oral Medicine Section, Department of Surgical Science, CIR Dental School, University of Turin, Turin, Italy

${ }^{10}$ Department of Medicine and Surgery, Oral Medicine and Laser Surgery Unit, University Center of Dentistry, University of Parma, Parma, Italy

${ }^{11}$ Department of Medical and Surgical Specialities, Radiological Sciences and Public Health, University of Brescia, Brescia, Italy

${ }^{12}$ Department of Surgical, Oncological, and Oral Sciences, University of Palermo, Palermo, Italy

${ }^{13}$ Department of Biomedical, Surgical and Dental Sciences, Maxillo-facial and Dental Unit, Fondazione IRCCS Ca' Granda Ospedale Maggiore Policlinico. University of Milan, Milan, Italy

${ }^{14}$ Department of Oncology, Oral Medicine and Oral Oncology Unit, University of Turin, Turin, Italy

${ }^{15}$ Department of Medical, Surgical and Health Sciences, University of Trieste, Trieste, Italy

${ }^{16}$ Unit of Oral and Maxillofacial Surgery, Ca' Foncello Hospital, Treviso, Italy

${ }^{17}$ Department of Oral Sciences and Maxillofacial Surgery, University of Rome La Sapienza, Rome, Italy

${ }^{18}$ Head and Neck Department, Fondazione Policlinico Universitario A. Gemelli IRCCS, School of Dentistry, Università Cattolica del Sacro Cuore, Rome, Italy

${ }^{19}$ Department of Economics and Statistics, University Federico II of Naples, Naples, Italy
}

Correspondence

Elena Calabria, Department of Neurosciences, Reproductive and Odontostomatological Sciences, University Federico II of Naples, Via Pansini no 5 Naples; 80131 Italy.

Email: calabriaelena92@gmail.com

\begin{abstract}
Objectives: To analyze psychological profiles, pain, and oral symptoms in patients with oral lichen planus (OLP).

Materials and methods: 300 patients with keratotic OLP (K-OLP; reticular, papular, plaque-like subtypes), 300 patients with predominant non-keratotic OLP (nK-OLP;
\end{abstract}




\section{Funding information}

The work was supported by the Department of Neurosciences, Reproductive and Odontostomatological Sciences, Oral Medicine Unit, of the "Federico II University of Naples," Italy. erythematosus atrophic, erosive, ulcerative, bullous subtypes), and 300 controls were recruited in 15 universities. The number of oral sites involved and oral symptoms were recorded. The Numeric Rating Scale (NRS), Total Pain Rating Index (T-PRI), Hamilton Rating Scales for Depression and for Anxiety (HAM-D and HAM-A), Pittsburgh Sleep Quality Index (PSQI), and Epworth Sleepiness Scale (ESS) were administered.

Results: The OLP patients, especially the nK-OLP, showed higher scores in the NRS, T-PRI, HAM-D, HAM-A and PSQI compared with the controls ( $p$-value $<.001^{* *}$ ). A positive correlation between the NRS, T-PRI, HAM-A, HAM-D, and PSQI was found with the number of oral symptoms and number of oral sites involved. Pain was reported in $67.3 \%$ of nK-OLP and $49.7 \%$ of K-OLP cases with poor correspondence between the site of lesions and the site of the symptoms.

Conclusions: Mood disorders are frequently associated with OLP with an unexpected symptomatology correlated with the number of oral symptoms and with the extension of disease suggesting a peripheral neuropathy.

\section{KEYWORDS}

anxiety, depression, oral lichen planus, pain, sleep disturbance

\section{1 | INTRODUCTION}

Oral lichen planus (OLP) is an immune-mediated inflammatory chronic disease of the oral mucosa, with an unknown etiology (DeAngelis et al., 2019) and a global prevalence of 1.01\% (González Moles et al. 2020). The association of several psychological comorbidities, such as anxiety (A), depression (D), and sleep disturbance $(S D)$, has been reported and should be evaluated in the patient's clinical management (Rojo-Moreno et al., 1998; Chaudhary, 2004; Lundqvist et al., 2006; Gavic et al. 2014, Adamo et al. 2015; Wiriyakijja et al., 2020a,b,c). OLP may have different patterns of clinical manifestations which range from keratotic manifestations (white reticular, papular and/or plaque-like lesions) to predominantly nonkeratotic lesions (atrophic, erythematous and/or erosive, ulcerative or bullous lesions) (Alrashdan et al., 2016; Carrozzo, et al., 2019). Furthermore, clinical variants can change over time in the same patient.

Keratotic OLP (K-OLP) is usually asymptomatic while predominantly non-Keratotic OLP (nK-OLP) is associated to oral discomfort, burning sensation, and even severe pain (Alberdi-Navarro et al.; 2020; Park et al.; 2012). Painful lesions may affect eating, swallowing, and speaking, causing an impaired quality of life in OLP patients (Wiriyakijja et al., 2020a,b,c). Moreover, other complex oral symptoms, probably related to changes in the surface of the oral mucosa, have been reported (Adamo et. al, 2017; Larsen et al., 2017). This symptomatology, in association with an unpredictable behavior of the disease and the risk of cancer development, could contribute to an impaired psychological well-being, causing emotional changes and a worsening of the quality of life of patients, which in time could amplify the symptoms of pain (Radwan-Oczko et al. 2018; VilarVillanueva et al. 2019; Wiriyakijja et al., 2020a,b,c).

It is also necessary to consider that pre-existing mood disorders may affect the perception of pain and the ability of the patient to cope with the disease and its symptoms, contributing not only to the development but also an exacerbation of the disease (Mohamadi Hasel et al., 2013).

Until now, a few single-center studies have evaluated the prevalence of mood disorders in OLP (Adamo et al. 2015; Chaitanya et al., 2020; Manczyk et al. 2019; Vallejo et al. 2001; Wiriyakijja et al 2020; Wiriyakijja et al 2020), analyzing the differences between K-OLP and nK-OLP, with unclear results (Rojo-Moreno et al. 1998), and no evident relationships between intensity and quality of pain (Larsen et al. 2017).

Recently, a high prevalence of A, D, and SD in a group of patients with K-OLP has been identified (Adamo et al. 2017; Alberdi-Navarro et al. 2020). In addition, this subset of patients was strongly symptomatic (Adamo et al. 2018) with a prevalent burning as an oral complaint in addition to mood disorders (Adamo et al. 2017).

Therefore, we decided to perform a multicenter case-control study in a large cohort of patients with different OLP clinical patterns in order to better evaluate the potential relationships between pain, burning sensations, and mood disorders in this disease. To the best of our knowledge, this is the first study which assesses oral symptoms and mood disorders in such a large number of patients with OLP.

The primary aim of this study was to analyze the psychological profile ( $A, D$, and $S D$ ), the reported pain and additional intraoral symptoms in cohorts of patients with K-OLP and nK-OLP, comparing 
the results between the two groups and with those relative to the control group.

The secondary objective of the present study was to analyze the topographic correlation between the site of the pain and the location of the OLP lesions and to investigate the correlation between the intensity and quality of the pain with the demographic factors, psychological profile, number of oral symptoms reported and number of sites of OLP lesions involved.

\section{2 | MATERIAL AND METHODS}

\section{1 | Study design}

This was an observational multicenter case-control study carried out in fifteen Italian Oral Medicine outpatients' departments of different Italian Universities (eight northern, three central, and four southern universities) join with the Italian Society of Oral Pathology and Medicine (SIPMO- Società Italiana di Patologia e Medicina Orale). The Oral Medicine Department of Federico II University of Naples was the chief investigator center and the Ethics Committee of the Federico II University of Naples approved the study (reference number: 184/18). All the other Oral Medicine departments which participated at the study had to obtain the ethical approval by their local ethics committees. The study was conducted in accordance with the ethical principles of the World Medical Association Declaration of Helsinki.

\section{2 | Participants}

Overall, the study groups comprised a total of $300 \mathrm{~K}-\mathrm{OLP}$ patients and 300 nK-OLP patients selected for the OLP group, and 300 healthy subjects for the control group.

The participants' recruitment was conducted between December 2018 and January 2020 and was based upon a convenience sampling. All potentially eligible participants in all Oral Medicine outpatients' clinics were invited to participate at the present study and provided their written informed consent.

Recruitment process ended when each department had included a total of twenty patients with K-OLP, twenty patients with nK-OLP and twenty healthy control subjects. The patients and controls were matched by age and gender. Firstly, we recruited the patients and then calculated the gender distribution and the average age; secondly, we recruited the controls to obtain matched sample.

Participants of either gender and aged 18 or older were included based upon the following inclusion/exclusion criteria. The inclusion criteria for the K-OLP and nK-OLP were as follows: (a) a clinical and histopathological confirmation of OLP based upon the modified WHO diagnostic criteria (van der Meij \& van der Waal, 2003); (b) patients with exclusive keratotic pattern (presence of white reticular, papular and/or plaque-like lesions) of the oral cavity were included in the K-OLP group; (c) patients with a predominant non-keratotic pattern and the occurrence of atrophic, erythematous, erosive, ulcerative, and /or bullous lesions with or without the presence of keratotic lesions were included in the nK-OLP group.

The exclusion criteria for both the K-OLP and nK-OLP groups were as follows: (a) pregnant or breastfeeding women; (b) evidence of oral epithelial dysplasia; (c) a suspicion that the oral lesions may be related to any drug use or oral restoration; (d) the presence of any other identified oral mucosal disease, autoimmune disease, serious systemic disease or tumor, or a history or occurrence of psychiatric illness as defined by the American Psychiatric Association Diagnostic and Statistical Manual of Mental Disorders 5 (DSM-5); (e) a history of alcohol or substance abuse; (f) ongoing treatment with systemic and/or topical corticosteroids or with psychotropic drugs; (g) an inability to understand the questionnaires.

The inclusion criteria for the control group encompassed: (a) patients referred to the same University Dental Clinics for routinely dental care during the study period; (b) the absence of any oral mucosal lesions. The exclusion criteria for the healthy controls were as follows: (a) pregnant or breastfeeding women; (b) the presence of any autoimmune disease, serious systemic disease or tumor or a history or occurrence of psychiatric illness as defined by the DSM-5; (c) a history of alcohol or substance abuse; (d) ongoing treatment with a psychotropic drug; (e) an inability to understand the questionnaires.

\section{3 | Sample size calculation}

The sample size, namely 300 subjects for each of the three groups, was set by fixing a test power of no less than $90 \%$ associated with a significance of no more than $5 \%$ (software G*Power 3.1.9.7 by Dusseldorf University). This sample size calculation was performed using the effect size estimation from a previous published research study regarding scales of mood disorders and pain (Adamo et al.,2015, 2017).

\section{$2.4 \mid$ Procedure}

All the patients underwent a comprehensive intra- and extra-oral examination carried out by two oral medicine experts with a documented experience in the evaluation and treatment of OLP.

At admission, sociodemographic data such as age, gender, educational level (in years), marital status, job status, social habits (smoking and alcohol consumption), oral mucosal disease onset, body mass index (BMI), the presence of any systemic diseases, and drug consumption were recorded for the patients and controls. Participants were given a set of questionnaires evaluating the intensity and quality of pain, their psychological status (level of anxiety and depression), and quality of sleep.

The questionnaires comprised:

1. the Numeric Rating Scale (NRS) and Total Pain Rating Index (T-PRI) from the Short Form of the McGill Pain Questionnaire 
(SF-MPQ) for the assessment of the oral discomfort, and the intensity and quality of pain (Hjermstad et al. 2011; Melzack, 1987);

2. the Hamilton Rating Scale for Depression (HAM-D) and the Hamilton Rating Scale for Anxiety (HAM-A) for the evaluation of depression and anxiety (Hamilton, 1959; Hamilton, 1960);

3. the Pittsburgh Sleep Quality Index (PSQI) and the Epworth Sleepiness Scale (ESS) for the assessment of sleep (Curcio et al.; 2013; Vignatelli et al. 2003).

All the questionnaires were reviewed for completeness before collection and were administered in their Italian versions. The present study was reported in accordance with the Strengthening the Reporting of Observational Studies in Epidemiology (STROBE) guidelines for observational studies (Appendix) (von Elm et al., 2014).

\section{5 | Outcomes}

The outcomes evaluated for the primary objective of the present study were the presence of psychological comorbidities as measured by HAM-A, HAM-D, PSQI, ESS, and the reported pain as measured by NRS, T-PRI along with additional intraoral symptoms, with the aim to detect any potential differences between the OLP subgroups and the control group. For the secondary objective, we recorded the location of the reported pain in each patient and the location of the OLP lesions in order to investigate the topographic correlation between the site of the pain and the location of the OLP lesions. Finally, we looked at the correlation between the intensity and quality of the pain with the psychological profile, number of intraoral symptoms reported and number of sites of OLP lesions involved. To determine associated predictors of psychological profile and pain, the clinical type of OLP (K-OLP versus nK-OLP) the number of oral symptoms and the number of oral sites were also assessed. In addition, independent predictors of worse pain were investigated. Demographic factors (gender, age, education, family status, and employment), current smoker, alcohol use (at least $\leq 14$ units/week) and body mass index, psychological factors (anxiety, depression and sleep disturbance), and clinical types of OLP (K-OLP and nK-OLP) were considered.

\section{6 | Outcome measures}

\subsection{1 | Measures of oral pain}

The Numeric Rating Scale (NRS-11) is a well-validated instrument for the evaluation of pain intensity whose scale ranges from 0 to $10(0=$ no oral symptoms and $10=$ the worst imaginable discomfort). Respondents are asked to report pain intensity in the last $24 \mathrm{hr}$ (Hjermstad et al. 2011).

The Total Pain Rating Index (T-PRI) from the short form of the McGill Pain Questionnaire (SF-MPQ) is a measure of the quality of pain and it is a multidimensional pain questionnaire which measures the sensory, affective, and evaluative aspects of the perceived pain. It comprises 15 items from the original MPQ, each scored from 0 (none) to 3 (severe). The T-PRI score is obtained by summing the item scores (range 0-45). There are no established critical cut points for the interpretation of the scores, and as for the MPQ, a higher score indicates worse pain (Melzack, 1987).

\subsection{2 | Measures of psychological factors}

The Hamilton Rating Scale for Anxiety (HAM-A) is a measure of anxiety symptoms and it comprises 14 items. Scores can range from 0 to 56 . A score $<17$ indicates mild symptoms, scores between 18 and 24 indicate mild-to-moderate severity, and scores $>25$ indicate moderate- to-severe anxiety (Hamilton, 1959).

The Hamilton Rating Scale for Depression (HAM-D) is a measure of depression symptoms and it comprises 21 items pertaining to the affective field. Scores can range from 0 to 54 . A score $>7$ indicates impairment. Scores in the 7-17 range indicate mild depression, scores between 18 and 24 indicate moderate depression, and scores over 24 indicate severe depression (Hamilton, 1960).

The Pittsburgh Sleep Quality Index (PSQI) is a standardized questionnaire assessing sleep quality and disturbances. This instrument comprises 19 items, generating 7 "component" scores: subjective sleep quality, sleep latency, sleep duration, habitual sleep efficiency, sleep disturbances, use of sleep medication, and daytime dysfunction. Each item is scored from 0 to 3 , with higher scores indicating poorer sleep or more frequent sleep problems. Items are combined to yield the seven components (scores ranging from 0 to 3 ), and the sum of the scores for these seven components yields 1 global score ranging from 0 to 21 . Global scores above five distinguish poor sleepers from good sleepers with high sensitivity (90\%-99\%) and specificity (84\%-87\%) (Curcio et al., 2013).

The Epworth Sleepiness Scale (ESS) measures subject's general level of daytime sleepiness. The instrument comprises 8 items assessing the propensity for sleep in eight common situations. Subjects rate their likelihood of dozing in each situation on a scale of 0 (would never doze) to 3 (a high chance of dozing). The ESS score is the sum of the eight items, ranging from 0 to 24 , with a cutoff value of $>10$ indicating excessive daytime sleepiness (Vignatelli et al. 2003).

\section{7 | Clinical assessment}

The participants were categorized into two groups considering the clinical variant of OLP (K-OLP vs. nK-OLP). The sites of oral mucosa involved by OLP lesions were also recorded. In addition, the clinicians analyzed the type and number of oral symptoms reported by the patients and the number of oral sites involved for both groups.

The oral symptoms reported were pain (often expressed with a predominant burning component, xerostomia, dysgeusia, sialorrhea, subjective halitosis, globus pharyngeus, itching sensation, intraoral 
foreign body sensation, tingling, occlusal dysesthesia, changes in the tongue morphology, oral dyskinesia and dysosmia (qualitative alteration or distortion of the perception of smell). A point was assigned to the presence of each of these symptoms, with a maximum of 13 .

The oral cavity was divided into nine sites: the maxillary gingiva, the mandibular gingiva, the right buccal mucosa, the left buccal mucosa, the upper/lower lips, the ventrum and dorsum of the tongue, the floor of the mouth, the hard palate, the soft palate. The upper and lower lips and the ventrum and dorsum of the tongue were considered each as one site. A point was assigned to the presence of OLP lesions at these sites, with a maximum of 9.

\section{8 | Statistical analysis}

The statistical analysis was performed using the SPSS software v. 23. Descriptive statistics, including means, standard deviations, medians, and interquartile range (IQR), were used to analyze the sociodemographic and clinical characteristics of the three groups. The Pearson Chi Square test was used to test the significance differences between the percentages in the three groups. Differences associated with $p$ values less than 0.05 or 0.01 were considered moderately or strongly significant, respectively. The non-parametric ANOVA procedure by Kruskal-Wallis was employed to test for any differences between the recorded medians of the HAM-D, HAM-A, PSQI, ESS, NRS, and T-PRI in the groups. $p$-values $<0.05$ were considered to reflect a statistical significance. The Mann-Whitney $U$ test with the Bonferroni correction was performed among the same variables in the three groups in any case in which a significant difference in the former test was found. In this analysis, $p$-values $<0.008$ were considered to represent a statistical significance. The Spearman test was used to analyze the correlation between the number of oral symptoms and the number of oral sites in relation to the NRS, T-PRI, HAM-A, HAM-D, PSQI, and ESS.

To identify potential predictors of intensity and quality of pain in $\mathrm{K}$ OLP and in nK-OLP patients, multiple linear regression analyses were performed, in each of the two OLP groups, considering sociodemographic variables, smoking, alcohol use, BMI, and psychological profile. Full models, when all the variables were entered simultaneously, were used to evaluate the relative contributions of these variables to pain.

In detail, a sequential regression model analysis including predictors, one by one, to obtain unadjusted coefficient estimations was performed. Moreover, in a final step, we performed a full model analysis considering all predictors, simultaneously, to estimate adjusted coefficients. In all steps, we provided standard errors of model coefficients which measure the statistical precision of inference estimation of the model parameters.

\section{3 | RESULTS}

A total of 900 participants, with no missing data recorded, were finally enrolled in the present study: $300 \mathrm{nK}$-OLP patients, $300 \mathrm{~K}$ OLP patients for the study groups, and 300 healthy subjects for the control group. The sociodemographic characteristics of the K-OLP and nK-OLP patients and controls are summarized in Table 1. A statistically significant difference was found in relation to education and BMI between the patients and controls ( $p$-values $<0.001^{* *}$ and $0.041^{*}$ respectively). In addition, the controls were characterized by a significantly higher percentage of employment (155 patients; $51.7 \%$ ) and a higher percentage of smokers (96 patients; $32.0 \%$ ).

Table 2 shows the frequency of systemic comorbidities and the drugs used by the patients of the study groups. Hypertension was the most common associated disease for patients and controls, and a statistically significant difference between patients and controls was found ( $p$-value $<0.001^{* *}$ ). A statistically significant difference was also found in relation to Beta-Adrenergic receptor blocker, Calcium channel blocker, ACE-inhibitor, Antiplatelet and Proton Pump Inhibitor use between patients and controls. Beta-Adrenergic receptor blockers, ACE-inhibitors, and Proton Pump Inhibitors were the most frequently taken drugs by the K-OLP and nK-OLP patients.

The clinical parameters and the multiple comparison test of the K-OLP and nK-OLP patients and controls are summarized in Table 3. NRS, T-PRI, HAM-A, HAM-D, and PSQI scores showed a statistically significant difference between the OLP patients and controls ( $p$-value $<0.001^{* *}$ ) but not with respect to the ESS ( $p$-value 0.315$)$. In addition, a significant statistical difference in relation to the NRS, T-PRI, and HAM-D scores ( $p$-value $<0.001^{* *} ;<0.001^{* *}$ and $<0.07^{*}$ respectively) between the K-OLP and nK-OLP groups was observed, but this was not the case in relation to the HAM-A and PSQI scores.

The score analysis of the psychological profiles of the K-OLP and nK-OLP patients has shown that $48.3 \%$ (145) of the K-OLP patients and 54\% (162) of the nK-OLP patients had A (HAM-A > 7); $44.7 \%$ (134) of the K-OLP patients and $51.3 \%$ (154) of nK-OLP patients had D (HAM-D > 7) and 49\% (147) of the K-OLP patients and 51.7\% (156) of the nK-OLP patients had SD (PSQI > 5). No statistically significant difference was detected between the two groups with regard to the scores rates distribution.

Table 4 shows the frequency of the oral symptoms, the oral sites involved by OLP lesions, the location of symptoms for the KOLP and nK-OLP groups, and the comparison test of the number of oral symptoms reported and the number of oral sites associated with pain/burning among the K-OLP and nK-OLP groups. For most of these patients, pain was described as burning in character. There was a statistically significant difference between the two groups in terms of pain/burning sensation ( $p$-value $<0.001^{* *}$ ) and sialorrhea ( $p$-value: $\left.0.004^{* *}\right)$, these two symptoms being the most frequently reported in nK-OLP. Burning was the most frequent and worst oral symptom reported, and present in 58.3\% (174) of the K-OLP patients and $71.7 \%(215)$ of the nK-OLP patients. The most frequently affected site involved in relation to the pain/burning was the tongue for the K-OLP patients (72.6\%) and the buccal mucosa for the nKOLP patients (90.6\%). In addition, the K-OLP patients more frequently reported a diffusion of the burning to the entire oral mucosa, compared with the nK-OLP patients (15.3\% vs. $8.7 \%, p$-value: 0.012 ).

From an analysis of these data, it emerges that the frequency of pain/burning was higher than the frequency of the oral sites 


\begin{tabular}{|c|c|c|c|c|}
\hline Demographic variables & K-OLP & nK-OLP & Controls & $p$-value \\
\hline Gender & Frequency (\%) & Frequency (\%) & Frequency (\%) & 1.000 \\
\hline Male & $125(41.7 \%)$ & $125(41.7 \%)$ & $125(41.7 \%)$ & \\
\hline Female & $175(58.3 \%)$ & $175(58.3 \%)$ & $175(58.3 \%)$ & \\
\hline \multirow[t]{2}{*}{ Age (in years) } & Mean $\pm S D$ & Mean $\pm S D$ & Mean $\pm S D$ & .686 \\
\hline & $65.2 \pm 12.2$ & $64.6 \pm 12.6$ & $64.2 \pm 16.9$ & \\
\hline \multirow[t]{2}{*}{ Education (in years) } & Mean $\pm S D$ & Mean $\pm S D$ & Mean $\pm S D$ & $<.001^{* *}$ \\
\hline & $10.9 \pm 4.0$ & $11.0 \pm 4.4$ & $13.6 \pm 4.5$ & \\
\hline Family situation & Frequency (\%) & Frequency (\%) & Frequency (\%) & $<.001^{* *}$ \\
\hline Single & 37 (12.3\%) & $27(9.0 \%)$ & $82(27.3 \%)$ & \\
\hline Married & 217 (72.3\%) & 209 (69.7\%) & $176(58.7 \%)$ & \\
\hline Divorced & $16(5.3 \%)$ & $14(4.7 \%)$ & $24(8.0 \%)$ & \\
\hline Widowed & 30 (10.0\%) & $50(16.7 \%)$ & $18(6.0 \%)$ & \\
\hline Employment & Frequency (\%) & Frequency (\%) & Frequency (\%) & $<.001^{* *}$ \\
\hline Employed & $108(36.0 \%)$ & $80(26.7 \%)$ & 155 (51.7\%) & \\
\hline Unemployed & $113(37.7 \%)$ & $158(52.7 \%)$ & $68(22.7 \%)$ & \\
\hline Retired & 79 (26.3\%\%) & $62(20.7 \%)$ & $77(25.7 \%)$ & \\
\hline \multirow[t]{2}{*}{ Body Mass Index } & Mean $\pm S D$ & Mean $\pm S D$ & Mean $\pm S D$ & $.041^{*}$ \\
\hline & $24.9 \pm 3.9$ & $25.0 \pm 4.0$ & $24.3 \pm 3.6$ & \\
\hline \multirow[t]{2}{*}{ Disease onset (years) } & Mean $\pm S D$ & Mean $\pm S D$ & Mean $\pm S D$ & $.020^{*}$ \\
\hline & $4.5 \pm 2.3$ & $4.3 \pm 2.7$ & NA & \\
\hline Risk factors & & & & $p$-value \\
\hline Smoker & Frequency (\%) & Frequency (\%) & Frequency (\%) & $<.001^{* *}$ \\
\hline Yes & $66(22.0 \%)$ & 52 (17.3\%) & 96 (32.0\%) & \\
\hline No & 234 (78.0\%) & 248 (82.7\%) & 204 (68.0\%) & \\
\hline Alcohol use & Frequency (\%) & Frequency (\%) & Frequency (\%) & .552 \\
\hline $\begin{array}{l}\text { Yes ( } \leq 14 \text { units/ } \\
\text { week) }\end{array}$ & 91 (30.3\%) & 83 (27.7\%) & 95 (31.7\%) & \\
\hline Not & 209 (69.7\%) & 217 (72.3\%) & 205 (68.3\%) & \\
\hline
\end{tabular}

TABLE 1 Sociodemographic profile, body mass index, disease onset, and risk factors in the $300 \mathrm{~K}-\mathrm{OLP}, 300 \mathrm{nK}-\mathrm{OLP}$ patients, and 300 controls

Note: IQR is the interquartile range. The significance difference between medians was measured by the Kruskal-Wallis test. "Significant $0.01<p \leq .05,{ }^{* *}$ Significant $p \leq .01$.

The Significance difference between the percentages was measured by the Pearson Chi Square test. "Significant $0.01<p \leq .05,{ }^{* *}$ Significant $p \leq .01$.

Abbreviations: ESS, Epworth sleepiness scale; HAM-A, Hamilton rating scale for anxiety; HAM-D, Hamilton rating scale for depression; K-OLP, keratotic oral lichen planus; nK-OLP, non-keratotic oral lichen planus; NRS, numeric rating scale; PSQI, Pittsburgh sleep quality index; T-PRI, total pain rating index.

involved by OLP lesions and that these symptoms were reported in sites without any clinical manifestation of the disease in each patient group. Therefore, there may be a poor topographic relationship between the location of the OLP lesions and the location of pain. In oral sites with a lower frequency of OLP lesions, such as the floor of the mouth (15.3\% of the K-OLP patients and $22.7 \%$ of the nK-OLP patients) and the soft palate (15.3\% of the K-OLP patients and $20.3 \%$ of the nK-OLP patients), the subjective perception of pain was found in $58.8 \%$ and $73.9 \%$ of the K-OLP patients, and $54.1 \%$ and $71.7 \%$ of the $\mathrm{nK}-\mathrm{OLP}$ patients respectively.

Other frequent symptoms reported were xerostomia (33.7\% of the K-OLP patients and $34.7 \%$ of the nK-OLP patients), dysgeusia (19.3\% of the K-OLP patients and $22.0 \%$ of the nK-OLP patients), sialorrhea $(10.3 \%$ of the K-OLP patients $18.7 \%$ of the nK-OLP patients), and subjective halitosis (18.3\% of the K-OLP and $20.3 \%$ of the nK-OLP patients).

Relatively uncommon symptoms reported were globus $(13.3 \%$ of the K-OLP patients and $22.0 \%$ of the nK-OLP patients), itching (11.4\% of the K-OLP patients and $22.0 \%$ of the nK-OLP patients), a tingling sensation $(9.7 \%$ of the K-OLP patients and $12.7 \%$ of the $\mathrm{nK}-$ OLP patients), occlusal dysesthesia (7.7\% of the K-OLP patients and $12.7 \%$ of the nK-OLP patients), and dysosmia (6.3\% of the K-OLP patients and of the nK-OLP patients).

The most frequent site involved by OLP lesions was the buccal mucosa, considering the right and left side for both groups (in $46.3 \%$ of the K-OLP patients and $45.8 \%$ of the nK-OLP patients). There was a 
TABLE 2 Frequency of systemic diseases and drug consumption in the $300 \mathrm{~K}-\mathrm{OLP}$ and $300 \mathrm{nK}-\mathrm{OLP}$ patients and 300 controls

\begin{tabular}{|c|c|c|c|c|}
\hline \multirow[b]{2}{*}{ Systemic disease } & \multicolumn{3}{|c|}{ Frequency (\%) } & \multirow[b]{2}{*}{$p$-value } \\
\hline & K-OLP & $\begin{array}{l}\text { nK- } \\
\text { OLP }\end{array}$ & Controls & \\
\hline Essential Hypertension & $32.7 \%$ & $48.0 \%$ & $26.0 \%$ & $<.001^{* *}$ \\
\hline Hypercholesterolemia & $22.3 \%$ & $23.0 \%$ & $16.7 \%$ & .109 \\
\hline $\begin{array}{l}\text { Previous myocardial } \\
\text { infarction }\end{array}$ & $2.0 \%$ & $2.3 \%$ & $2.7 \%$ & .864 \\
\hline Diabetes & $8.3 \%$ & $9.3 \%$ & $7.0 \%$ & .762 \\
\hline Asthma & $2.3 \%$ & $5.7 \%$ & $2.3 \%$ & $.035^{*}$ \\
\hline $\begin{array}{l}\text { Gastro-esophageal } \\
\text { reflux disease }\end{array}$ & $15.3 \%$ & $21.3 \%$ & $9.0 \%$ & $<.001^{* *}$ \\
\hline Hepatitis B & $1.3 \%$ & $0.7 \%$ & 0.0 & .134 \\
\hline Hepatitis C & $3.3 \%$ & $3.3 \%$ & $1.3 \%$ & .214 \\
\hline Endocrine disease & $3.7 \%$ & $5.3 \%$ & $2.0 \%$ & .094 \\
\hline Hypothyroidism & $11.3 \%$ & $10.7 \%$ & $7.0 \%$ & .154 \\
\hline Hyperthyroidism & $1.7 \%$ & $3.7 \%$ & $1.3 \%$ & .111 \\
\hline $\begin{array}{l}\text { Benign prostatic } \\
\text { hypertrophy }\end{array}$ & $7.0 \%$ & $6.0 \%$ & $2.75 \%$ & $.044^{*}$ \\
\hline $\begin{array}{l}\text { Previous malignant } \\
\text { disease }\end{array}$ & $8.0 \%$ & $8.0 \%$ & $5.3 \%$ & .341 \\
\hline \multicolumn{5}{|l|}{ Drug Consumption } \\
\hline $\begin{array}{l}\text { Beta-Adrenergic } \\
\text { receptor blockers }\end{array}$ & $15.7 \%$ & $19.3 \%$ & $11.7 \%$ & $.001^{* *}$ \\
\hline $\begin{array}{l}\text { Angiotensin II receptor } \\
\text { blockers }\end{array}$ & $8.0 \%$ & $8.3 \%$ & $5.7 \%$ & .394 \\
\hline Diuretics & $8.0 \%$ & $8.3 \%$ & $8.0 \%$ & .985 \\
\hline $\begin{array}{l}\text { Calcium Channel } \\
\text { blockers }\end{array}$ & $4.7 \%$ & $9.3 \%$ & $3.7 \%$ & $.006^{* *}$ \\
\hline ACE-inhibitors & $9.3 \%$ & $19.7 \%$ & $10.3 \%$ & $<.001^{* *}$ \\
\hline Simvastatin & $14.3 \%$ & $19.3 \%$ & $13.7 \%$ & .115 \\
\hline Metformin & $8.0 \%$ & $6.7 \%$ & $5.3 \%$ & .424 \\
\hline Insulin & $2.7 \%$ & $2.7 \%$ & $2.0 \%$ & .830 \\
\hline Antiplatelets & $11.7 \%$ & $16.0 \%$ & $8.0 \%$ & $.010^{* *}$ \\
\hline Blood thinner & $5.0 \%$ & $4.7 \%$ & $2.0 \%$ & .114 \\
\hline Levothyroxine sodium & $12 \%$ & $12 \%$ & $6.3 \%$ & $.029 *$ \\
\hline Proton pump inhibitors & $14.0 \%$ & $19.7 \%$ & $11.7 \%$ & $<.001^{*}$ \\
\hline
\end{tabular}

Note: The significance difference between percentages was measured by the Pearson Chi Square test. *Significant $0.01<p \leq .05,{ }^{* *}$ Significant $p \leq .01$.

Abbreviations: K-OLP, keratotic oral lichen planus; nK-OLP, nonkeratotic oral lichen planus.

statistically significant difference in the location of the lesions on the floor of the mouth between the K-OLP and nK-OLP groups ( $p$-value: $0.023^{*}$ ).

Moreover, a statistically significant difference was observed in the number of oral symptoms reported by the K-OLP and nK-OLP patients ( $p$-value: $0.006^{* *}$ ) with the latter group reporting a greater number of oral symptoms, but not in terms of the number of oral sites involved ( $p$-value 0.412).
Table 5 shows a positive correlation between the total scores of the NRS (pain intensity), T-PRI (pain quality), HAM-A (anxiety), HAM-D (depression), and PSQI (sleep disturbance) with the number of oral symptoms reported and the number of oral sites involved for the K-OLP and nK-OLP groups.

The results of the simultaneous multiple linear regression analyses for the K-OLP and nK-OLP groups, predicting the intensity and quality of pain (NRS and T-PRI), are shown in Table 6. The model tests the contribution of the demographic variables and confounding factors with pain. The first model tests the contributions of the demographic variables and habits to the intensity and quality of pain (NRS and T-PRI) which were not found to be statistically significant for either the K-OLP or nK-OLP groups. The addition of $A$ (model 2 ) resulted in a significant increase in the R2 value for NRS (K-OLP: DR2 = 8.1\%; $p$-value < $0.001^{* *}$; nK-OLP: DR2 $=6.4 \% ; p$-value $\left.<0.001^{* *}\right)$ and for T-PRI (K-OLP: DR2 = 4.3\%; $p$-value $0.003^{*}$; nK-OLP: DR2 $=19.8 \% ; p$-value $<0.001^{* *}$ ). The addition of $D$ (model 3 ) resulted in a significant increase in the R2 value for NRS (K-OLP: DR2 $=4.6 \%$; $p$-value < $0.001^{* *}$; nK-OLP: DR2 $=3.5 \% ; p$-value $\left.0.001^{* *}\right)$ and for T-PRI (K-OLP: DR2 = 4.1\%; $p$-value < 0.001**; nK-OLP: DR2 $=15.9 \% ; p$-value $\left.<0.001^{* *}\right)$. The addition of $S D$ (model 4 ) resulted in a significant increase in the R2 value for NRS (K-OLP: DR2 = 9.2\%; $p$-value < $0.001^{* *}$; nK-OLP: DR2 = 7.7\%; $p$-value $0.001^{* *}$ ) and for T-PRI (K-OLP: DR2 = 2.9\%; $p$-value 0.002**; nK-OLP: DR2 = 10.6\%; $p$-value $\left.<0.001^{* *}\right)$. The final full model (model 5 ) in which all of the variables were entered simultaneously could explain $11.5 \%, 4.6 \%, 10.4 \%$, and $21.2 \%$ of the variance in the total NRS score (pain intensity) and total T-PRI score (quality of pain), respectively, for the K-OLP and nK-OLP groups.

\section{4 | DISCUSSION}

The present study provides an evaluation of psychological profile and oral symptoms in a large group of patients with different OLP clinical patterns.

The association of psychological factors with OLP and their role with respect to this condition continue to be frequently underestimated despite several studies have suggested that stress, anxiety, depression, and sleep disturbances could affect and impair the patient's quality of life, and contribute to the onset and exacerbation of the disease (Adamo et al. 2015; Alves et al., 2015; Manczyk et al. 2019; Vallejo et al. 2001; Vilar-Villanueva et al. 2019; Wiriyakijja et al., 2020a,b,c).

Similar to these considerations, a recent meta-analysis of Jalenques et al showed a prevalence of depression and anxiety in $27 \%$ and in $28 \%$, respectively, of patients affected by lichen planus (Jalenques et al. 2020). Similarly, Wiriyakijia et al found a prevalence of anxiety and depression of $39.3 \%$ and $20.77 \%$, respectively, in 260 OLP patients and concluded that anxiety was the second most common comorbidity in OLP patients after hypertension (Wiriyakijja et al., 2020a,b,c). These findings were close with the results of the study from Gavic et al. (2014). 


\begin{tabular}{|c|c|c|c|c|}
\hline \multirow[b]{2}{*}{ Clinical parameters } & \multirow{2}{*}{$\frac{\text { K-OLP }}{\text { Median; IQR }}$} & \multirow{2}{*}{$\frac{\text { nK-OLP }}{\text { Median; IQR }}$} & \multirow{2}{*}{$\frac{\text { Controls }}{\text { Median; IQR }}$} & \multirow[b]{2}{*}{$p$-value } \\
\hline & & & & \\
\hline NRS & 2.0; [0-5] & $4.0 ;[0.3-6]$ & $0.0 ;[0-0]$ & $<.001^{* *}$ \\
\hline T-PRI & 2.0; [0-5] & $3.0 ;[1-7]$ & $0.0 ;[0-0]$ & $<.001^{* *}$ \\
\hline HAM-A & 7.0; [3-12] & 8.0; [4-15] & 5.0; [1-10] & $<.001^{* *}$ \\
\hline HAM-D & 6.0; [3-12] & 8.0; [4-13] & $5.0 ;[2-9]$ & $<.001^{* *}$ \\
\hline PSQI & $5.0 ;[3-8]$ & 6.0; [4-9] & 5.0; [3-7] & $<.001^{* *}$ \\
\hline ESS & 4.5.0; [2-8] & 5.0; [2-8] & $5.0 ;[3-8]$ & .315 \\
\hline \multicolumn{5}{|c|}{ Multiple comparison test } \\
\hline & & K-OLP & nK-OLP & \\
\hline \multirow[t]{3}{*}{ NRS } & K-OLP & ------ & & \\
\hline & nK-OLP & $<0.001^{*}$ & ------ & \\
\hline & Controls & $<0.001^{*}$ & $<0.001^{*}$ & \\
\hline \multirow[t]{3}{*}{ T-PRI } & K-OLP & $-\cdots--$ & & \\
\hline & nK-OLP & $<0.001^{*}$ & ----- & \\
\hline & Controls & $<0.001^{*}$ & $<0.001^{*}$ & \\
\hline \multirow[t]{3}{*}{ HAM-A } & K-OLP & ----- & & \\
\hline & nK-OLP & 0.009 & ----- & \\
\hline & Controls & $<0.001^{*}$ & $<0.001^{*}$ & \\
\hline \multirow[t]{3}{*}{ HAM-D } & K-OLP & ------ & & \\
\hline & nK-OLP & $0.007^{*}$ & ----- & \\
\hline & Controls & $<0.001^{*}$ & $<0.001^{*}$ & \\
\hline \multirow[t]{3}{*}{ PSQI } & K-OLP & ------ & & \\
\hline & nK-OLP & 0.107 & ----- & \\
\hline & Controls & $<0.004^{*}$ & $<0.001^{*}$ & \\
\hline
\end{tabular}

TABLE 3 Clinical parameters and multiple comparison test of the NRS, T-PRI, HAM-A, HAM-D, PSQI, and ESS in the K-OLP, nK-OLP patients and the control group

Note: IQR is the interquartile range. The significance difference between medians was measured by the Kruskal-Wallis test. ${ }^{*}$ Significant $0.01<p \leq .05,{ }^{* *}$ Significant $p \leq .01$.

Multiple comparison test using the Mann-Whitney $\mathrm{U}$ test with the Bonferroni correction. ${ }^{*}$ Test is significant with a $p$-value $<.008$

Abbreviations: ESS, Epworth sleepiness scale; HAM-A, Hamilton rating scale for anxiety; HAM-D, Hamilton rating scale for depression; K-OLP, keratotic oral lichen planus; nK-OLP, non-keratotic oral lichen planus; NRS, numeric rating scale; PSQI, Pittsburgh sleep quality index; T-PRI, total pain rating index.

The results of the present research further confirm the higher prevalence of anxiety and depression, but also of sleep disturbances, in OLP patients compared with healthy subjects. Until now, this is the first case-control study examining the prevalence of mood disorders in such a large group of patients with OLP. In the OLP patients considered, we found anxiety in 51\% (307 out of 600 patients), depression in 48\% (291 out of 600), and SD in 50.5\% (303 out of 600). An analysis of multiple comparisons revealed that the nK-OLP pattern was associated with a higher frequency of depression compared with K-OLP while no differences in the prevalence of anxiety and sleep disturbances were found between the two groups. Therefore, the prevalence of mood disorders in OLP was definitely higher compared with the results of the previous studies and mood disorders should be considered one of the most prevalent comorbidities in OLP patients. In general, these results confirm the higher prevalence of anxiety in OLP compared with other psychological factors such as depression, as also previously described (Girardi et al., 2011; Liao et al., 2020; Zucoloto et al. 2019).

Considering the differences in the clinical variants of OLP, we found a higher prevalence of depression in nK-OLP patients compared with K-OLP patients, in contrast with other previous studies (Lundqvist et al. 2006; Lundqvist et al. 2006; Rojo-Moreno et al., 1998; Vallejo et al. 2001) that reported a higher prevalence of both psychological comorbidities in erosive OLP.

In this study, a comprehensive analysis of painful oral symptoms was carried out, considering that pain is the most important reason encouraging OLP patients to seek treatment from a clinician (Gupta et al., 2017; Lodi et al. 2012). In our study, the OLP patients reported a great frequency of oral discomfort (64.3\%; 389 patients), mostly referred as burning sensation, with the nK-OLP patients reporting a higher prevalence and significantly greater level of pain compared with the K-OLP patients. Most of these patients reported a long 
TAB LE 4 Frequency of oral symptoms, oral sites involved, and location of burning in the $300 \mathrm{~K}-\mathrm{OLP}$ and $300 \mathrm{nK}-\mathrm{OLP}$ patients and comparison test of the number of oral symptoms reported and the number of oral sites involved

\begin{tabular}{|c|c|c|c|}
\hline Oral symptoms & K-OLP Frequency (\%) & nK-OLP Frequency (\%) & $p$-value \\
\hline Pain/Burning localized in all sites & $15.3 \%$ & $8.7 \%$ & $.012^{*}$ \\
\hline Dysgeusia & $19.3 \%$ & $22.0 \%$ & .420 \\
\hline Sialorrhea & $10.3 \%$ & $18.7 \%$ & $.004^{* *}$ \\
\hline Globus pharyngeus & $13.3 \%$ & $17.0 \%$ & .211 \\
\hline Itching & $11.4 \%$ & $12.7 \%$ & .637 \\
\hline Intraoral Foreign Body Sensation & $11.7 \%$ & $12.7 \%$ & .708 \\
\hline Tingling sensation & $9.7 \%$ & $12.7 \%$ & .243 \\
\hline Dysosmia & $6.3 \%$ & $6.3 \%$ & 1.000 \\
\hline Oral sites involved by OLP & K-OLP Frequency (\%) & nK-OLP Frequency (\%) & $p$-value \\
\hline Upper Gingiva & $21.3 \%$ & $19.6 \%$ & .407 \\
\hline Lower Gingiva & $21.3 \%$ & $19.6 \%$ & .407 \\
\hline Lips & $24.0 \%$ & $29.3 \%$ & .140 \\
\hline Right Buccal mucosa & $23.1 \%$ & $23 \%$ & .900 \\
\hline Left Buccal mucosa & $23.1 \%$ & $23 \%$ & .900 \\
\hline Tongue & $39.7 \%$ & $41.3 \%$ & .678 \\
\hline Floor of the Mouth & $15.3 \%$ & $22.7 \%$ & $.023^{*}$ \\
\hline Right Buccal mucosa & $37 \%$ & $45.3 \%$ & $<.001^{* *}$ \\
\hline Left Buccal mucosa & $36.5 \%$ & $45 \%$ & $<.001^{* *}$ \\
\hline Tongue & $72.6 \%$ & $87.4 \%$ & $.004^{* *}$ \\
\hline Floor of the Mouth & $58.8 \%$ & $73.9 \%$ & .098 \\
\hline Hard Palate & $63.3 \%$ & $81.6 \%$ & $.009^{* *}$ \\
\hline Soft Palate & $54.1 \%$ & $71.7 \%$ & .063 \\
\hline \multicolumn{4}{|l|}{ Comparison test } \\
\hline & K-OLP & nK-OLP & \\
\hline & Median; IQR & Median; IQR & $p$-value \\
\hline $\mathrm{N}^{\circ}$ oral symptoms & 1.0; [1-3] & $2.0 ;[1-3]$ & $.006^{* *}$ \\
\hline $\mathrm{N}^{\circ}$ oral sites & $2.0 ;[1-3]$ & $2.0 ;[1-3]$ & .412 \\
\hline
\end{tabular}

Note: The significance difference between the percentages was measured by the Pearson Chi Square test. ${ }^{*}$ Significant $.01<p \leq .05,{ }^{* *}$ Significant $p \leq .01$.

The significance difference between the medians was measured by the Mann-Whitney $\mathrm{U}$ test. ${ }^{*}$ Significant $.01<p \leq .05,{ }^{* *}$ Significant $p \leq .01$. Abbreviations: K-OLP, keratotic oral lichen planus; nK-OLP, non-keratotic oral lichen planus.

duration of continuous pain/burning present daily, worsening with sour or spicy food. It is interesting to note that 174 (58.3\%) K-OLP patients, a cluster generally asymptomatic, reported pain/ burning symptoms diffuse in the entire oral mucosa in $15.3 \%$ (45) of cases. 
TAB LE 5 Correlation analysis between the number of oral symptoms reported, the number of oral sites involved and the NRS, T-PRI, HAM-A, HAM-D, PSQI, and ESS scores in the $300 \mathrm{~K}-\mathrm{OLP}$ and $300 \mathrm{nK}-\mathrm{OLP}$ patients

\begin{tabular}{|c|c|c|c|c|}
\hline & K-OLP & & nK-OLP & \\
\hline Clinical parameters & $N^{\circ}$ oral symptoms & $N^{\circ}$ oral sites & $N^{\circ}$ oral symptoms & $N^{\circ}$ oral sites \\
\hline TPRI & $0.472\left(<0.001^{* *}\right)$ & $0.348\left(<0.001^{* *}\right)$ & $0.535\left(<0.001^{* *}\right)$ & $0.351\left(<0.001^{* *}\right)$ \\
\hline HAM-A & $0.261\left(<0.001^{* *}\right)$ & $0.224\left(<0.001^{* *}\right)$ & $0.343\left(<0.001^{* *}\right)$ & $0.153\left(0.008^{* *}\right)$ \\
\hline PSQI & $0.293\left(<0.001^{* *}\right)$ & $0.184\left(<0.001^{* *}\right)$ & $0.300\left(<0.001^{* *}\right)$ & $0.201\left(0.001^{* *}\right)$ \\
\hline
\end{tabular}

Note: The significance correlations were measured by the Spearman correlation test. ${ }^{*}$ Significant $.01<p \leq .05,{ }^{* *}$ Significant $p \leq .01$.

Abbreviations: HAM-A, Hamilton rating scale for anxiety; HAM-D, Hamilton rating scale for depression; K-OLP, keratotic oral lichen planus; nK-OLP, non-keratotic oral lichen planus; PSQI, Pittsburgh sleep quality index.

However, there was a poor topographic relationship between location of the OLP lesions and the reported pain/burning, which was also described in sites without OLP lesions in both groups. This seems to suggest that in many cases, pain is not correlated with the site of lesions. In addition to pain, many K-OLP patients presented several complex additional symptoms, some of which have never previously been reported, such as occlusal dysesthesia, oral dyskinesia, and dysosmia (qualitative alteration or distortion of the perception of smell).

The median number of oral symptoms was higher for the nKOLP patients while no differences in terms of the extension of the disease (number of sites involved by OLP lesions) were found between the two groups. The correlation analysis revealed that anxiety, depression, sleep disturbances, and the intensity and quality of pain/burning were positively correlated with the number of oral symptoms and with the number of oral sites involved. Therefore, there was an association between a higher number of symptoms and a higher number of sites involved by OLP lesions and higher scores in anxiety, depression, and sleep disturbances, also with a greater subjective perception in the intensity and quality of pain. However, the quality and intensity of pain were poorly correlated with mood disorders suggesting that the perception of pain seems to be an independent factor, related to the extension rather than the severity of the disease. Indeed, an analysis of the last model of the hierarchical regression (model 5) suggests that the sociodemographic variables, habits, anxiety, depression, and sleep disturbances could explain only $11.5 \%$ and $8.9 \%$ of the variance in pain intensity for the K-OLP and nK-OLP patients respectively, and only $10.4 \%$ and $21.2 \%$ of the variance in quality of pain for the K-OLP and nK-OLP patients, respectively. These results may indicate that pain and mood are processed somewhat independently in the central nervous system, as suggested in imaging studies (Lee et al.,2011; Martucci \& Mackey, 2018).

These findings are relatively new and in contrast with previous studies where OLP patients with anxiety and depression reported a higher intensity of oral pain compared with patients with a normal psychological status, suggesting that mood disorders may amplify the perception of the pain (Adamo et al. 2015; Wiriyakijja et al., 2020a,b,c), or with the study of Liao et al. where the anxiety was considered as a starting point of the disease possibly occurring prior to the OLP onset and modifying the subjective perception of the patients (Liao et al 2019). However, we cannot exclude the possibility that in a long-standing disease, the simultaneous presence of untreated mood disorders and the stress associated with the potentially malignant evolution of the disease could contribute to an amplification of the pain perception. (Mohamadi et al., 2013).

In previous studies, we identified a subset of patients with $\mathrm{K}$ OLP who were strongly symptomatic with a condition resembling that of burning mouth syndrome and were successfully treated with antidepressants (Adamo et al.,2015, 2017). Therefore, we concluded that some OLP patients may simultaneously present with another disease (Adamo et al. 2018). However, from the results of the current study it is not possible to exclude the hypothesis of a common etiopathogenesis which involves an imbalance in the central and peripheral nervous systems.

The central nervous system and the immunological processes are strictly and bidirectionally interconnected through neurotransmitters, hormones, and cytokines, as demonstrated by psychoneuroimmunology (Kusnecov et al., 1989). Recent studies have suggested that significant immune disturbances in the periphery may concomitantly influence the immune state of the brain, which in turn could modify the immune modulation peripheral pathways, contributing to the susceptibility of the subject to autoimmune disorders, particularly in genetically predisposed individuals (Dantzer, 2018; Theofilopoulos et al., 2017).

OLP is a chronic inflammatory immunological disease in which a key role is played by the increase in local pro-inflammatory cytokines, such as tumor necrosis factor-alpha (TNF- $\alpha$ ), interleukin-1 (IL1), interleukin-6 (IL-6), interleukin-8 (IL-8), interleukin-10 (IL-10), and interleukin 17 (IL-17), released by the endothelial cells, macrophages and keratinocytes and contributing to the recruitment of activated T-lymphocytes (Capuron \& Miller, 2011; Carvalho et al. 2019; Lu et al. 2015; Solimani et al. 2019; Wei et al. 2018). The T-lymphocytes and pro-inflammatory cytokine in the oral submucosa have a critical 


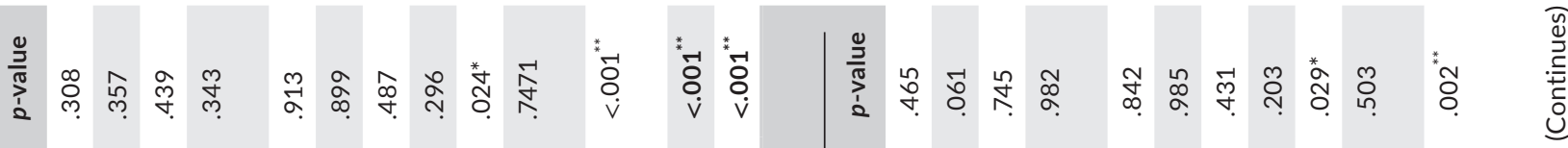

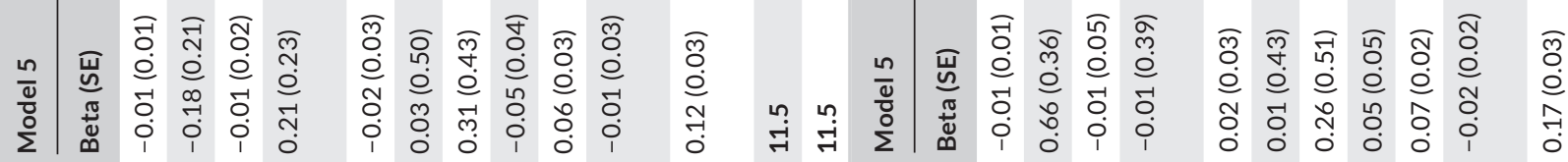

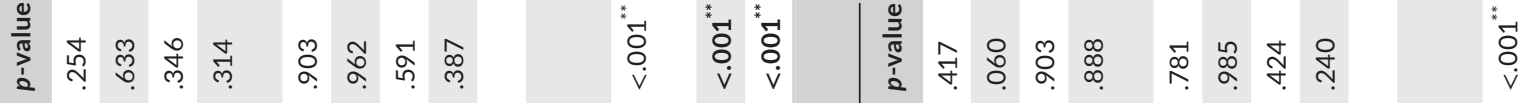

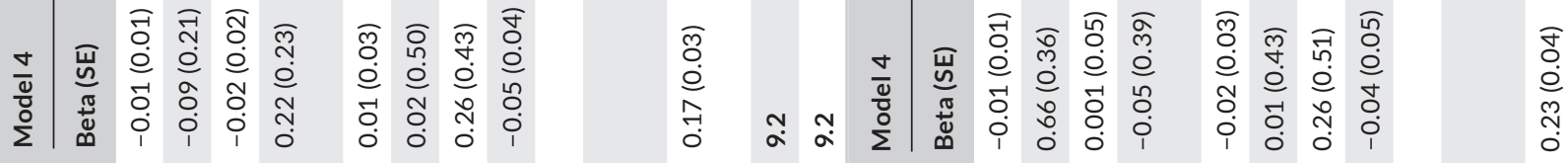

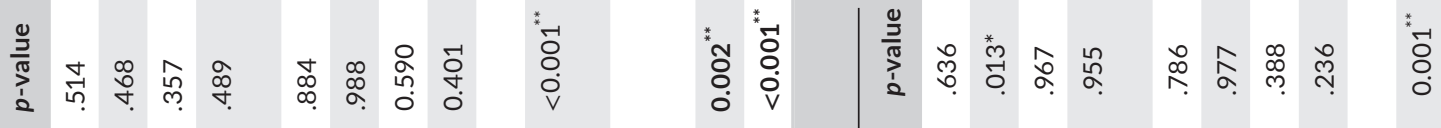

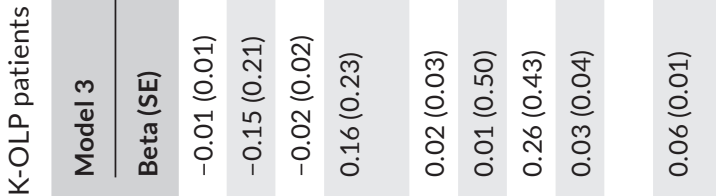

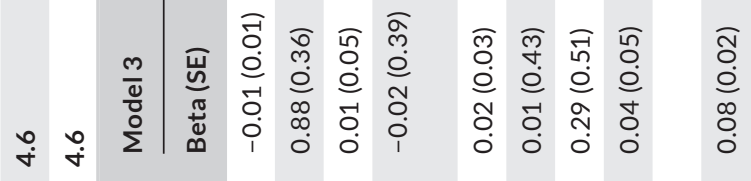

이

몬

$\stackrel{\overrightarrow{0}}{\stackrel{0}{0}}$

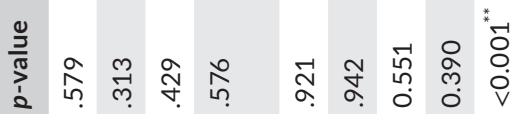

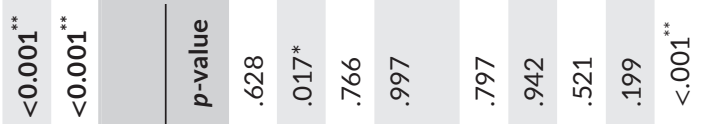

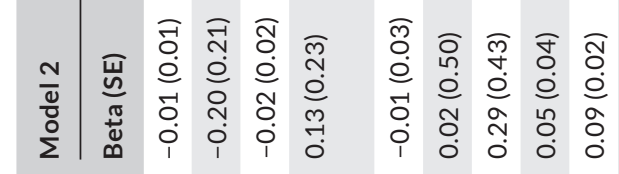

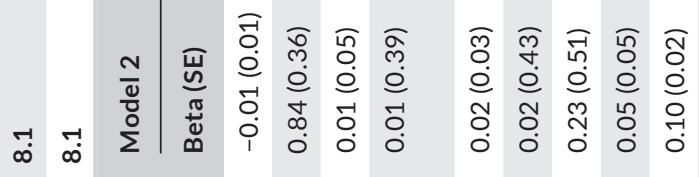

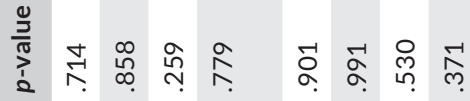

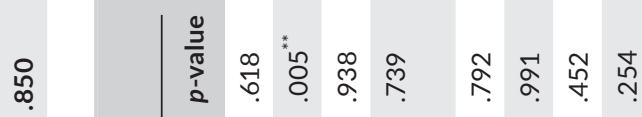

은

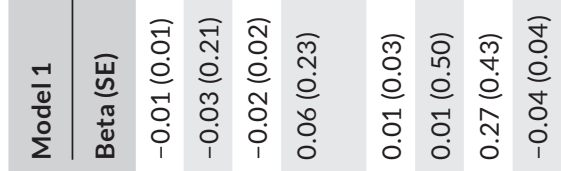

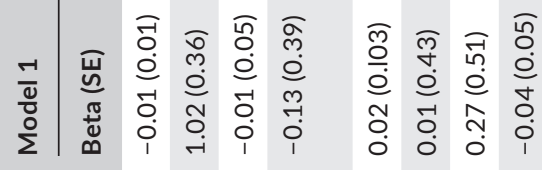

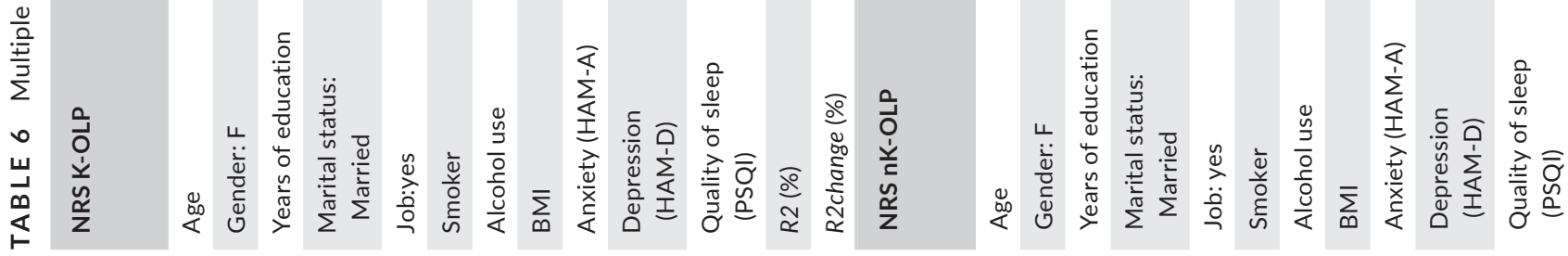




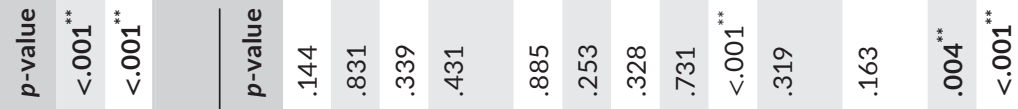

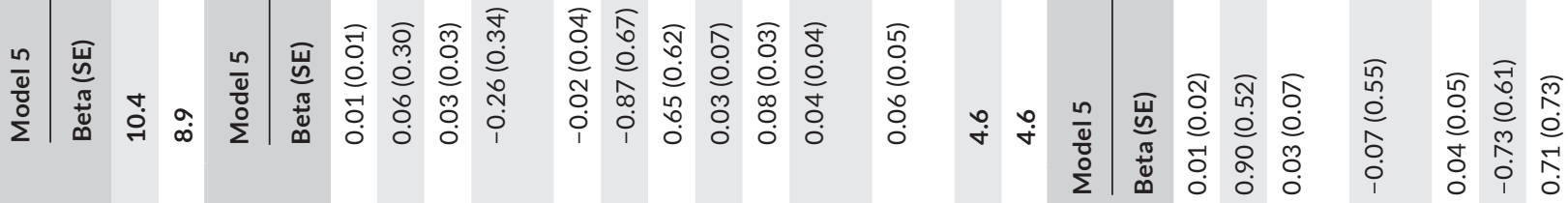

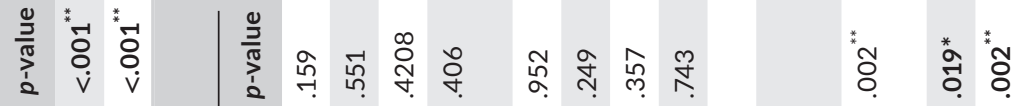

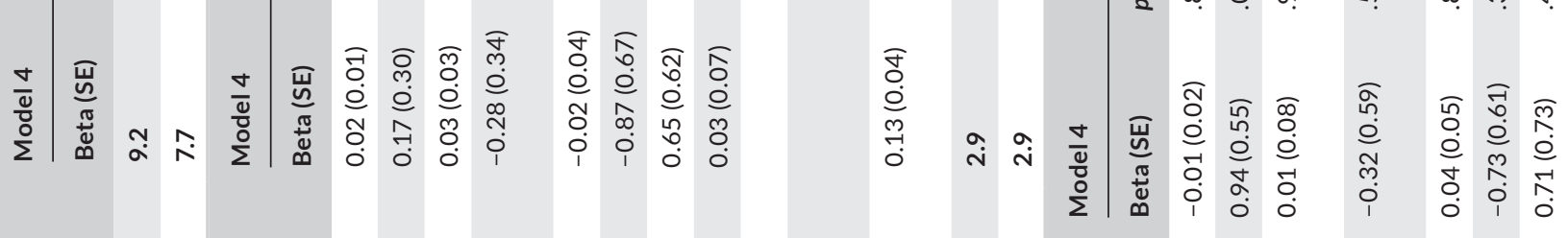

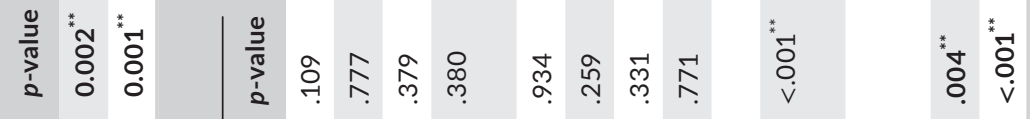

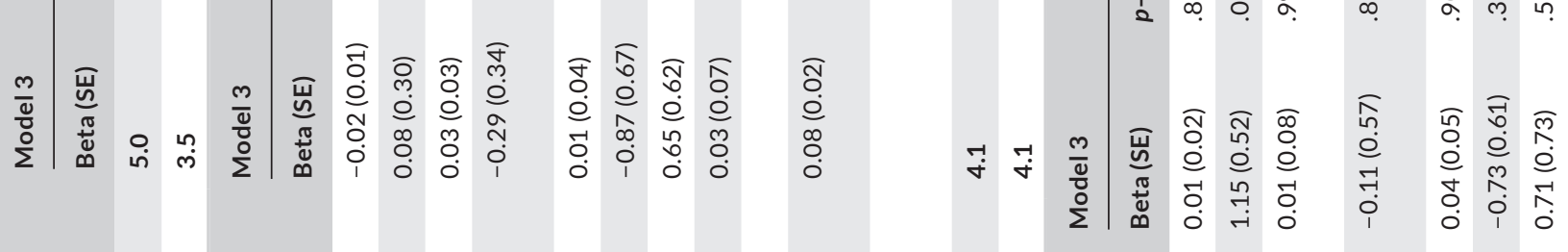

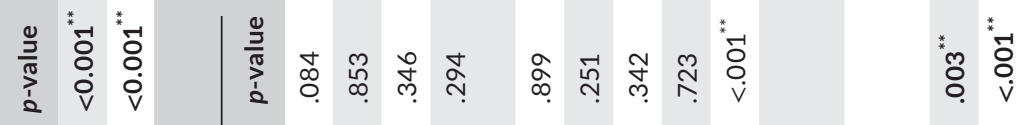

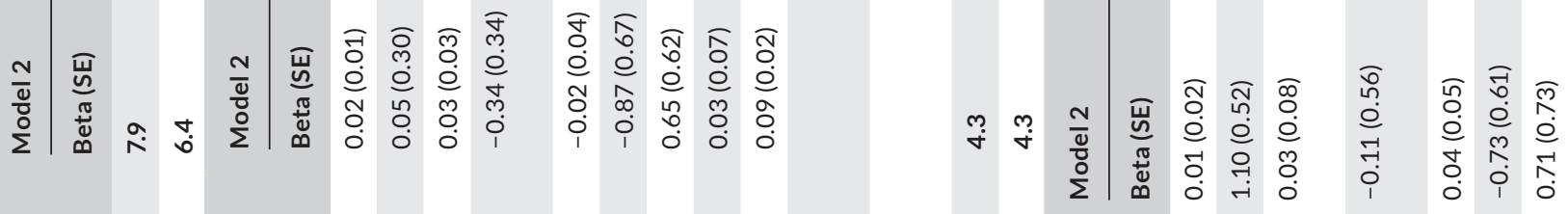

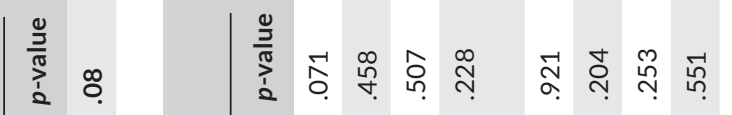

क्ष

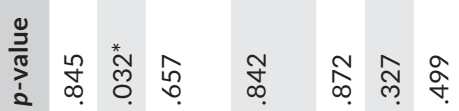

I

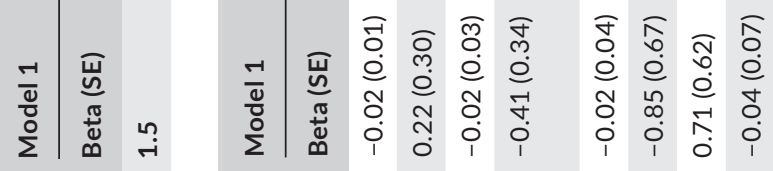

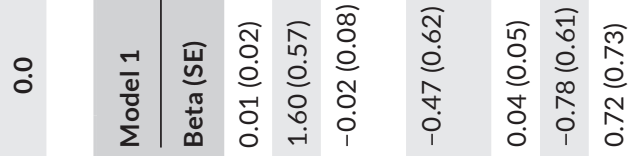

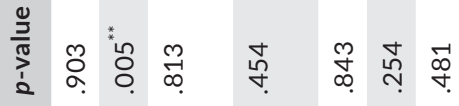

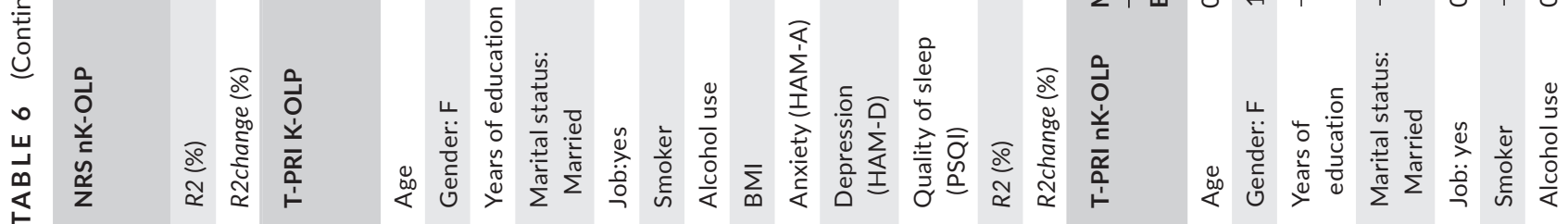


role in the pathogenesis of OLP, and in turn, may contribute to damage the unmyelinated nerve $C$ small fibers causing an increase in pain perception (Duksal et al. 2016; Magrinelli et al. 2015), in a similar way in which these molecules may participate in mutagenic processes leading to the transformation of normal cells into cancer cells (Anand et al., 2016; Mravec et al., 2020).

In addition, these pro-inflammatory cytokines can access the brain and could negatively modulate the descending pain systems (Shubayev et al. 2010). In this context, the simultaneous presence of mood disorders could additionally contribute to a worsening of this autoimmune disease (Liu \& Tang, 2018) through the suppression of the protective immunity, exacerbating the chronic inflammation and causing a further increase in the pro-inflammatory cytokines of the brain, such as IL-1, IL- 6 and IL-10. In turn, this could aggravate the pain perception over the time through the activation of the hypothalamic-pituitary-adrenal (HPA) axis and the sympathetic-adrenal axis induced by the neuroendocrine mediators (Kappelmann et al., 2020; Ratnayake et al. 2013). Furthermore, mood disorders seem to be associated with neuronal activation in some brain areas, such as the amygdalae and anterior insula, implicated in processing the motivational-affective /integrative dimension of pain (Giesecke et al. 2015; Namkung et al., 2018).

With a bidirectional relationship, the deficiencies in the activity of the endocrine system may contribute to the incidence of immunological diseases (Trombetta et al.; 2017). Indeed, the increase of certain pro-inflammatory hormones, such as cortisol, prolactin, and the estrogens, may cause the activation of the local proliferation and distribution of inflammatory cells, and subsequently the production of pro-inflammatory cytokines (Cutolo \& Straub, 2009; Girardi et al. 2011). Moreover, these hormonal changes may appear before the symptomatic phase of many autoimmune diseases, and therefore, it is not possible to exclude the possibility that the first step could be an alteration of the HPA axis, which may promote the loss of immunological tolerance which is a key feature of the etiopathogenesis of OLP (Bellavance \& Rivest, 2014; Jara et al. 2006).

Therefore, the results of the present study seem to suggest that the subjective perception of pain in some patients can be potentially due to a peripheral and/or central neuropathy, particularly in those patients with many sites involved by OLP lesions, independently of the mood disorders, which, if present, over time could further amplify the pain.

In the management of OLP, it is crucial to assess for the presence of mood disorders and pain using validated tools. Scales such as the NRS or Visual Analogic Scale (VAS), which evaluate only pain intensity, might not be sufficient to reflect the real impact of the symptoms in the patient's life. Therefore, clinicians should analyze not only the intensity of pain but also the psychological profile of their patients. They should consider the clinical subtypes of the OLP lesions, the extension of the disease in terms of the oral sites involved, the characteristics of the symptomatology of the pain including its quality and its location, the topographic relationship existing between the lesions and the symptoms, the frequency, duration and 
modifying factors of the oral discomfort as well as the additional oral symptoms in order to better understand the characteristics of the patients and plan an adequate treatment.

Until now, in the management of OLP, clinicians have assessed the disease activity based only upon the clinical oral presentation of the disease (Carrozzo et al., 2019; Escudier, et al. 2007). However, the results of this study suggest that patients with OLP show a complex symptomatology without a perfect correspondence between the clinical presentation and the experience and perception of the symptoms. Therefore, in symptomatic OLP patients for whom there is no topographic relationship between the location of the pain and the location of the OLP lesions, clinicians should consider a different pharmacological treatment, taking into account also the psychological profile of the patient. Currently, no curative treatment has been determined, and therefore, the primary management goal must be to relieve the symptoms of pain (Thongprasom, Carrozzo, Furness, \& Lodi, 2011). However, if anxiety, depression, and sleep disturbances are identified, the clinicians must take responsibility for requesting the assistance of other appropriate specialists, possibly in a multidisciplinary setting, in order to plan a suitable and comprehensive treatment for these patients.

\section{1 | Limitations}

The study has several limitations. The primary limitation is the absence of any evaluation of the disease activity in accordance with the clinical disease severity score system available in literature. The decision not to adopt this system was based on the evidence of recent studies (Zucoloto et al., 2019), where the severity of the clinical signs of OLP was found not to be a predictor of mood disorders and pain. In addition, we did not analyze the differences in age, sex, and psychological profile between the OLP patients with different clinical patterns. Similarly, we did not evaluate the differences between the symptom profiles and the scores of anxiety, depression, and sleep disturbances because it is plausible that the different symptom profiles might be associated with different psychological imbalances and their consequences.

Secondly, the results of the study might be considered exploratory and should be interpreted carefully, taking into account the cross-sectional design of the study and the fact that the analysis was made in tertiary referral Oral Medicine Units, with the presence of confounding factors due to the heterogeneity of the case-control study, particularly in multicenter settings.

Finally, it is not possible to establish a cause-effect relationship between pain and mood due the nature of the study design.

\section{5 | CONCLUSIONS}

This study has confirmed the higher prevalence of anxiety, depression, and sleep disturbances in OLP, with nK-OLP patients presenting a higher prevalence of depression symptoms and a significantly greater level of pain compared with K-OLP patients. In addition, patients with OLP, particularly nK-OLP patients, exhibit various and complex additional oral symptoms, frequently undetected.

This unexpected and subjective symptomatology in OLP patients seems to be strongly correlated with the number of additional oral symptoms reported and with the extension of the disease in terms of the number of oral sites involved by OLP lesions, and poorly with the psychological profile, suggesting a peripheral and central neuropathy in which mood and pain are interconnected but processed somewhat independently in the central nervous system.

A multidisciplinary setting is strongly advised for a proper management of patients with OLP in which clinicians of different medical specialties should not only focus on the treatment of the visible clinical signs of OLP alone but should screen patients carefully, including undertaking a comprehensive analysis of the psychological profile and oral symptoms.

They should use validated tools to screen mood disorders and to examine the topographic relationship between the symptoms of pain, the lesions and the extension of the disease in order to better identify patients who require additional treatment, including psychological support with conventional therapies. Further studies are required to confirm our results and to evaluate the causal relationships among these variables and the starting points.

\section{ACKNOWLEDGEMENTS}

DA, EC, NC, and MDM contributed for the conceptualization of the study, the methodology, the data collection and curation, and drafted the paper. MA analyzed the data and contributed in writing the manuscript. All the other authors were involved in the data collection and reviewed the manuscript. The authors have no conflict of interest to declare. The study received no external funding.

\section{COMPLIANCE WITH ETHICAL STANDARDS}

Explanation of any conflicts of interests: The authors have no conflict of interests to declare no financial support and no off-label or investigational use. The content of the manuscript has not been previously published and is not currently submitted elsewhere. All authors have contributed to the work and are familiar with the primary data; each has read the final version of the manuscript and approved its content. All authors have agreed to have their name added to the paper. The manuscript is free of falsification, fabrication, plagiarism, and self-plagiarism. All applicable principles of scientific integrity, including ethical treatment of human subjects, have been followed.

\section{AUTHOR CONTRIBUTIONS}

Daniela Adamo: Conceptualization; Data curation; Investigation; Methodology; Project administration; Writing-original draft; Writing-review \& editing. Elena Calabria: Conceptualization; Data curation; Investigation; Methodology; Project administration; Writing-original draft; Writing-review \& editing. Noemi Coppola: Conceptualization; Data curation; Investigation; Methodology; 
Project administration; Writing-original draft; Writing-review \& editing. Lorenzo Lo Muzio: Data curation; Writing-review \& editing. Michele Giuliani: Data curation; Writing-review \& editing. Maria Eleonora Bizzoca: Data curation; Writing-review \& editing. Lorenzo Azzi: Data curation; Writing-review \& editing. Fabio Croveri: Data curation; Writing-review \& editing. Giuseppe Colella: Data curation; Writing-review \& editing. Ciro Boschetti: Data curation; Writing-review \& editing. Lucio Montebugnoli: Data curation; Writing-review \& editing. DAVIDE BARTOLOMEO GISSI: Data curation; Writing-review \& editing. Mario Gabriele: Data curation; Writing-review \& editing. Marco Nisi: Data curation; Writing-review \& editing. Andrea Sardella: Data curation; Writingreview \& editing. Giovanni Lodi: Data curation; Writing-review \& editing. Elena Maria Maria Varoni: Data curation; Writing-review \& editing. Amerigo Giudice: Data curation; Writing-review \& editing. Alessandro Antonelli: Data curation; Writing-review \& editing. Marco Cabras: Data curation; Writing-review \& editing. Alessio Gambino: Data curation; Writing-review \& editing. Paolo Vescovi: Data curation; Writing-review \& editing. Alessandra Majorana: Data curation; Writing-review \& editing. Elena Bardellini: Data curation; Writing-review \& editing. Giuseppina Campisi: Data curation; Writing-review \& editing. Vera Panzarella: Data curation; Writingreview \& editing. Francesco Spadari: Data curation; Writing-review \& editing. Sonia Marino: Data curation; Writing-review \& editing. Monica Pentenero: Data curation; Writing-review \& editing. Marco Ardore: Data curation; Writing-review \& editing. Matteo Biasotto: Data curation; Writing-review \& editing. Margherita Gobbo: Data curation; Writing-review \& editing. Luca Guarda-Nardini: Data curation; Writing-review \& editing. Umberto Romeo: Data curation; Writing-review \& editing. Gianluca Tenore: Data curation; Writingreview \& editing. Rosario Serpico: Data curation; Writing-review \& editing. Carlo Lajolo: Data curation; Writing-review \& editing. Gioele Gioco: Data curation; Writing-review \& editing. Massimo Aria: Data curation; Formal analysis; Methodology; Project administration; Software; Writing-original draft; Writing-review \& editing. Michele Mignogna: Conceptualization; Data curation; Investigation; Methodology; Project administration; Supervision; Writing-original draft; Writing-review \& editing.

\section{INFORMED CONSENT}

For this type of study, written informed consent was obtained from all the patients included in the study.

\section{ETHICAL APPROVAL}

All procedures performed were in accordance with the ethical standards of the institutional and/or national research committee and with the 1964 Helsinki declaration and its later amendments or comparable ethical standards.

\section{PEER REVIEW}

The peer review history for this article is available at https://publo ns.com/publon/10.1111/odi.13787.

\section{ORCID}

Daniela Adamo (iD https://orcid.org/0000-0002-3784-4229

Elena Calabria (iD https://orcid.org/0000-0003-0739-5650

Lorenzo Azzi iD https://orcid.org/0000-0003-2532-7651

Andrea Sardella (iD https://orcid.org/0000-0003-4491-4640

Giovanni Lodi iD https://orcid.org/0000-0002-0218-8292

Amerigo Giudice iD https://orcid.org/0000-0002-5091-6979

Marco Cabras iD https://orcid.org/0000-0002-8240-5874

Elena Bardellini iD https://orcid.org/0000-0002-3955-4794

Monica Pentenero iD https://orcid.org/0000-0003-3972-1203

Gioele Gioco iD https://orcid.org/0000-0002-8637-2029

\section{REFERENCES}

Adamo, D., Cascone, M., Celentano, A., Ruoppo, E., Leuci, S., Aria, M., \& Mignogna, M. D. (2017). Psychological profiles in patients with symptomatic reticular forms of oral lichen planus: A prospective cohort study. Journal of Oral Pathology \& Medicine, 46(9), 810-816. https://doi.org/10.1111/jop.12577

Adamo, D., Mignogna, M. D., Pecoraro, G., Aria, M., \& Fortuna, G. (2018). Management of reticular oral lichen planus patients with burning mouth syndrome-like oral symptoms: A pilot study. The Journal of Dermatological Treatment, 29(6), 623-629. https://doi. org/10.1080/09546634.2018.1425359

Adamo, D., Ruoppo, E., Leuci, S., Aria, M., Amato, M., \& Mignogna, M. D. (2015). Sleep disturbances, anxiety and depression in patients with oral lichen planus: A case-control study. Journal of the European Academy of Dermatology and Venereology: JEADV, 29(2), 291-297. https://doi.org/10.1111/jdv.12525

Alberdi-Navarro, J., Aguirre-Urizar, J. M., \& Ginestal-Gómez, E. (2020). Clinical presentation of burning mouth syndrome in patients with oral lichenoid disease. Medicina Oral Patología Oral Y Cirugia Bucal, e805-e809. https://doi.org/10.4317/medoral.23812.

Alrashdan, M. S., Cirillo, N., \& McCullough, M. (2016). Oral lichen planus: A literature review and update. Archives of Dermatological Research, 308(8), 539-551. https://doi.org/10.1007/s00403-016-1667-2

Alves, M. G. O., do Carmo Carvalho, B. F., Balducci, I., Cabral, L. A. G., Nicodemo, D., \& Almeida, J. D. (2015). Emotional assessment of patients with oral lichen planus. International Journal of Dermatology, 54 , 29-32. https://doi.org/10.1111/ijd.12052

Anand, R., Sarode, G. S., \& Sarode, S. C. (2016). Chronic stress and oral cancer research: Disregarded aspects in animal model studies. Oral Oncology, 54, e5-e6. https://doi.org/10.1016/j.oraloncolo gy.2015.12.010

Bellavance, M. A., \& Rivest, S. (2014). The HPA - immune axis and the immunomodulatory actions of glucocorticoids in the brain. Frontiers in Immunology, 5, 136. https://doi.org/10.3389/fimmu.2014.00136

Capuron, L., \& Miller, A. H. (2011). Immune system to brain signaling: Neuropsychopharmacological implications. Pharmacology \& Therapeutics, 130(2), 226-238. https://doi.org/10.1016/j.pharm thera.2011.01.014

Carrozzo, M., Porter, S., Mercadante, V., \& Fedele, S. (2019). Oral lichen planus: A disease or a spectrum of tissue reactions? Types, causes, diagnostic algorhythms, prognosis, management strategies. Periodontology 2000, 80(1), 105-125. https://doi.org/10.1111/prd.12260.

Carvalho, M., Cavalieri, D., Do Nascimento, S., Lourenço, T., Ramos, D., Pasqualin, D., Martins, L., Rocha, F. A., Heller, D., \& Marti, L. (2019). Cytokines levels and salivary microbiome play a potential role in oral lichen planus diagnosis. Scientific Reports, 9(1), 18137. https://doi. org/10.1038/s41598-019-54615-y

Chaitanya, N. C., Reshmapriyanka, D., Pallavi, K., Ameer, S., Appala, A., Chowdhary, A., Prabhath, T., Ratna, M. P., Sowmya, B. S., Vaishnavi, 
C., \& Bontala, P. (2020). Serological and psychological assessment of patients with oral lichen planus using serum cortisol levels and hads questionnaire-a case control study. Journal of Population Therapeutics \& Clinical Pharmacology, 27(2), e19-e27. https://doi.org/10.15586/ jptcp.v27i2.667

Curcio, G., Tempesta, D., Scarlata, S., Marzano, C., Moroni, F., Rossini, P. M., Ferrara, M., \& De Gennaro, L. (2013). Validity of the Italian version of the Pittsburgh Sleep Quality Index (PSQI). Neurological Sciences, 34(4), 511-519. https://doi.org/10.1007/s10072-012-1085-y

Cutolo, M., \& Straub, R. H. (2009). Insights into endocrine-immunological disturbances in autoimmunity and their impact on treatment. Arthritis Research \& Therapy, 11(2), 218. https://doi.org/10.1186/ar2630

Dantzer, R. (2018). Neuroimmune interactions: From the brain to the immune system and vice versa. Physiological Reviews, 98(1), 477-504. https://doi.org/10.1152/physrev.00039.2016

DeAngelis, L. M., Cirillo, N., \& McCullough, M. J. (2019). The immunopathogenesis of oral lichen planus-Is there a role for mucosal associated invariant T cells? Journal of Oral Pathology \& Medicine, 48(7), 552-559. https://doi.org/10.1111/jop.12898

Duksal, T., Tiftikcioglu, B. I., Bilgin, S., Kose, S., \& Zorlu, Y. (2016). Role of inflammation in sensory neuropathy in prediabetes or diabetes. Acta Neurologica Scandinavica, 133(5), 384-390. https://doi.org/10.1111/ ane. 12474

Escudier, M., Ahmed, N., Shirlaw, P., Setterfield, J., Tappuni, A., Black, M. M., \& Challacombe, S. J. (2007). A scoring system for mucosal disease severity with special reference to oral lichen planus. The British Journal of Dermatology, 157(4), 765-770. https://doi. org/10.1111/j.1365-2133.2007.08106.x

Gavic, L., Cigic, L., Biocina Lukenda, D., Gruden, V., \& Gruden Pokupec, J. S. (2014). The role of anxiety, depression, and psychological stress on the clinical status of recurrent aphthous stomatitis and oral lichen planus. Journal of Oral Pathology \& Medicine, 43(6), 410-417. https:// doi.org/10.1111/jop.12148

Giesecke, T., Gracely, R. H., Williams, D. A., Geisser, M. E., Petzke, F. W., \& Clauw, D. J. (2005). The relationship between depression, clinical pain, and experimental pain in a chronic pain cohort. Arthritis and Rheumatism, 52(5), 1577-1584. https://doi.org/10.1002/art.21008

Girardi, C., Luz, C., Cherubini, K., de Figueiredo, M. A., Nunes, M. L., \& Salum, F. G. (2011). Salivary cortisol and dehydroepiandrosterone (DHEA) levels, psychological factors in patients with oral lichen planus. Archives of Oral Biology, 56, 864-868. https://doi.org/10.1016/j. archoralbio.2011.02.003

González-Moles, M. Á., Warnakulasuriya, S., González-Ruiz, I., GonzálezRuiz, L., Ayén, Á., Lenouvel, D., Ruiz-Ávila, I., \& Ramos-García, P. (2020). Worldwide prevalence of oral lichen planus: A systematic review and meta-analysis. Oral Diseases, https://doi.org/10.1111/odi.13323

Gupta, S., Ghosh, S., \& Gupta, S. (2017). Interventions for the management of oral lichen planus: A review of the conventional and novel therapies. Oral Diseases, 23(8), 1029-1042. https://doi.org/10.1111/ odi.12634

Hamilton, M. (1959). The assessment of anxiety states by rating. British Journal of Medical Psychology, 32(1), 50-55.

Hamilton, M. (1960). A rating scale for depression. Journal of Neurology, Neurosurgery \& Psychiatry. 23(1):56-62.

Hjermstad, M. J., Fayers, P. M., Haugen, D. F., Caraceni, A., Hanks, G. W., Loge, J. H., Fainsinger, R., Aass, N., \& Kaasa, S. (2011). Studies comparing Numerical Rating Scales, Verbal Rating Scales, and Visual Analogue Scales for assessment of pain intensity in adults: A systematic literature review. Journal of Pain and Symptom Management, 41(6), 1073-1093. https://doi.org/10.1016/j.jpainsymman.2010.08.016

Jalenques, I., Lauron, S., Almon, S., Pereira, B., D'Incan, M., \& Rondepierre, F. (2020). Prevalence and odds of signs of depression and anxiety in patients with Lichen Planus: Systematic review and meta-analyses. Acta Dermato-Venereologica, 100(18), adv00330-https://doi. org/10.2340/00015555-3660
Jara, L. J., Navarro, C., Medina, G., Vera-Lastra, O., \& Blanco, F. (2006). Immune-neuroendocrine interactions and autoimmune diseases. Clinical \& Developmental Immunology, 13(2-4), 109-123. https://doi. org/10.1080/17402520600877059

Kappelmann, N., Arloth, J., Georgakis, M. K., Czamara, D., Rost, N., Ligthart, S., Khandaker, G. M., \& Binder, E. B. (2020). Dissecting the association between inflammation, metabolic dysregulation, and specific depressive symptoms. JAMA Psychiatry, 78(2), 161-170. https://doi.org/10.1001/jamapsychiatry.2020.3436

Kusnecov, A., King, M. G., \& Husband, A. J. (1989). Immunomodulation by behavioural conditioning. Biological Psychology, 28(1), 25-39. https:// doi.org/10.1016/0301-0511(89)90109-9

Larsen, K. R., Johansen, J. D., Reibel, J., Zachariae, C., Rosing, K., \& Pedersen, A. (2017). Oral symptoms and salivary findings in oral lichen planus, oral lichenoid lesions and stomatitis. BMC Oral Health, 17(1), 103. https://doi.org/10.1186/s12903-017-0393-2

Lee, Y. C., Nassikas, N. J., \& Clauw, D. J. (2011). The role of the central nervous system in the generation and maintenance of chronic pain in rheumatoid arthritis, osteoarthritis and fibromyalgia. Arthritis Research \& Therapy, 13(2), 211. https://doi.org/10.1186/ar3306

Liao, H., Luo, Y., Long, L., Peng, J., Qiu, X., Yuan, P., Xu, H., \& Jiang, L. (2020). Anxiety and oral lichen planus. Oral Diseases, https://doi. org/10.1111/odi.13569. [Online ahead of print]

Liu, Y., \& Tang, X. (2018). Depressive syndromes in autoimmune disorders of the nervous system: Prevalence, etiology, and influence. Frontiers in Psychiatry, 9, 451. https://doi.org/10.3389/ fpsyt.2018.00451

Lodi, G., Carrozzo, M., Furness, S., \& Thongprasom, K. (2012). Interventions for treating oral lichen planus: A systematic review. The British Journal of Dermatology, 166(5), 938-947. https://doi. org/10.1111/j.1365-2133.2012.10821.x

Lu, R., Zhang, J., Sun, W., Du, G., \& Zhou, G. (2015). Inflammation-related cytokines in oral lichen planus: An overview. Journal of Oral Pathology \& Medicine, 44(1), 1-14. https://doi.org/10.1111/jop.12142

Lundqvist, E. N., Wahlin, Y. B., Bergdahl, M., \& Bergdahl, J. (2006). Psychological health in patients with genital and oral erosive lichen planus. Journal of the European Academy of Dermatology and Venereology: JEADV, 20, 661-666. https://doi. org/10.1111/j.1468-3083.2006.01559

Magrinelli, F., Briani, C., Romano, M., Ruggero, S., Toffanin, E., Triolo, G., Peter, G. C., Praitano, M., Lauriola, M. F., Zanette, G., \& Tamburin, S. (2015). The Association between serum cytokines and damage to large and small nerve fibers in diabetic peripheral neuropathy. Journal of Diabetes Research, 2015, 547834. https://doi. org/10.1155/2015/547834

Manczyk, B., Gołda, J., Biniak, A., Reszelewska, K., Mazur, B., Zając, K., Bińczak, P., Chomyszyn-Gajewska, M., \& Oruba, Z. (2019). Evaluation of depression, anxiety and stress levels in patients with oral lichen planus. Journal of Oral Science, 61(3), 391-397. https://doi. org/10.2334/josnusd.18-0113

Martucci, K. T., \& Mackey, S. C. (2018). Neuroimaging of pain: Human evidence and clinical relevance of central nervous system processes and modulation. Anesthesiology, 128(6), 1241-1254. https://doi. org/10.1097/ALN.0000000000002137

Melzack, R. (1987). The short-form McGill Pain Questionnaire. Pain, 30(2), 191-197. https://doi.org/10.1016/0304-3959(87)91074-8

Mohamadi Hasel, K., Besharat, M. A., Abdolhoseini, A., Alaei Nasab, S., \& Niknam, S. (2013). Relationships of personality factors to perceived stress, depression, and oral lichen planus severity. International Journal of Behavioral Medicine, 20(2), 286-292. https:// doi.org/10.1007/s12529-012-9226-5

Mravec, B., Tibensky, M., \& Horvathova, L. (2020). Stress and cancer. Part I: Mechanisms mediating the effect of stressors on cancer. Journal of Neuroimmunology, 346, 577311. https://doi.org/10.1016/j. jneuroim.2020.577311 
Namkung, H., Kim, S. H., \& Sawa, A. (2018). The Insula: An underestimated brain area in clinical neuroscience, psychiatry, and neurology. Trends in Neurosciences, 41(8), 551-554. https://doi.org/10.1016/j. tins.2018.05.004

Park, H. K., Hurwitz, S., \& Woo, S. B. (2012). Oral lichen planus: REU scoring system correlates with pain. Oral Surgery, Oral Medicine, Oral Pathology and Oral Radiology, 114(1), 75-82. https://doi. org/10.1016/j.oooo.2012.02.013

Radwan-Oczko, M., Zwyrtek, E., Owczarek, J. E., \& Szczesniak, D. (2018). Psychopathological profile and quality of life of patients with oral lichen planus. Journal of Applied Oral Science: Revista FOB, 26, e20170146. https://doi.org/10.1590/1678-7757-2017-0146

Ratnayake, U., Quinn, T., Walker, D. W., \& Dickinson, H. (2013). Cytokines and the neurodevelopmental basis of mental illness. Frontiers in Neuroscience, 7, 180. https://doi.org/10.3389/fnins.2013.00180

Rojo-Moreno, J. L., Bagan, J. V., Rojo-Moreno, J., Donat, J. S., Milian, M. A., \& Jimenez, Y. (1998). Psychologic factors and oral lichen planus. A psychometric evaluation of 100 cases. Oral Surgery, Oral Medicine, Oral Pathology, Oral Radiology, and Endodontics, 86, 687-691. https:// doi.org/10.1016/S1079-2104(98)90205-0.

Shubayev, V. I., Kato, K., \& Myers, R. R. Cytokines in pain, Chapter 8. In Kruger, L., \& A. Light (Eds.), Translational pain research: From mouse to man. : CRC Press/Taylor \& Francis.

Solimani, F., Pollmann, R., Schmidt, T., Schmidt, A., Zheng, X., Savai, R., Mühlenbein, S., Pickert, J., Eubel, V., Möbs, C., Eming, R., \& Hertl, M. (2019). Therapeutic targeting of Th17/Tc17 Cells leads to clinical improvement of Lichen Planus. Frontiers in Immunology, 10, 1808. https://doi.org/10.3389/fimmu.2019.01808

Theofilopoulos, A. N., Kono, D. H., \& Baccala, R. (2017). The multiple pathways to autoimmunity. Nature Immunology, 18(7), 716-724. https://doi.org/10.1038/ni.3731

Trombetta, A. C., Meroni, M., \& Cutolo, M. (2017). Steroids and autoimmunity. Frontiers of Hormone Research, 48, 121-132. https://doi. org/10.1159/000452911

Vallejo, M. J., Huerta, G., Cerero, R., \& Seoane, J. M. (2001). Anxiety and depression as risk factors for oral lichen planus. Dermatology (Basel, Switzerland), 203, 303-307. https://doi.org/10.1159/000051777

van der Meij, E. H., \& van der Waal, I. (2003). Lack of clinicopathologic correlation in the diagnosis of oral lichen planus based on the presently available diagnostic criteria and suggestions for modifications. Journal of Oral Pathology \& Medicine, 32(9), 507-512. https://doi. org/10.1034/j.1600-0714.2003.00125

Vignatelli, L., Plazzi, G., Barbato, A., Ferini-Strambi, L., Manni, R., Pompei, F., D'Alessandro, R., \& GINSEN (Gruppo Italiano Narcolessia Studio Epidemiologico Nazionale (2003). Italian version of the Epworth sleepiness scale: External validity. Neurological Sciences, 23(6), 295300. https://doi.org/10.1007/s100720300004

Vilar-Villanueva, M., Gándara-Vila, P., Blanco-Aguilera, E., Otero-Rey, E. M., Rodríguez-Lado, L., García-García, A., \& Blanco-Carrión, A.
(2019). Psychological disorders and quality of life in oral lichen planus patients and a control group. Oral Diseases, 25(6), 1645-1651. https://doi.org/10.1111/odi.13106

von Elm, E., Altman, D. G., Egger, M., Pocock, S. J., Gøtzsche, P. C., Vandenbroucke, J. P., \& Initiative, S. T. R. O. B. E. (2014). The Strengthening the Reporting of Observational Studies in Epidemiology (STROBE) Statement: Guidelines for reporting observational studies. International Journal of Surgery (London, England), 12(12), 1495-1499. https://doi.org/10.1016/j.ijsu.2014.07.013

Wei, W., Sun, Q., Deng, Y., Wang, Y., Du, G., Song, C., Li, C., Zhu, M., Chen, G., \& Tang, G. (2018). Mixed and inhomogeneous expression profile of Th1/Th2 related cytokines detected by cytometric bead array in the saliva of patients with oral lichen planus. Oral Surgery, Oral Medicine, Oral Pathology and Oral Radiology, 126(2), 142-151. https://doi.org/10.1016/j.oooo.2018.02.013

Wiriyakijja, P., Porter, S., Fedele, S., Hodgson, T., McMillan, R., Shephard, M., \& Ni Riordain, R. (2020). Health-related quality of life and its associated predictors in patients with oral lichen planus: A crosssectional study. International Dental Journal, https://doi.org/10.1111/ idj.12607. [In press]

Wiriyakijja, P., Porter, S., Fedele, S., Hodgson, T., McMillan, R., Shephard, M., \& Ni Riordain, R. (2020). Meaningful improvement thresholds in measures of pain and quality of life in oral lichen planus. Oral Diseases, https://doi.org/10.1111/odi.13379

Wiriyakijja, P., Porter, S., Fedele, S., Hodgson, T., McMillan, R., Shephard, M., \& Ni Riordain, R. (2020). Validation of the HADS and PSS-10 and psychological status in patients with oral lichen planus. Oral Diseases, 26(1), 96-110. https://doi.org/10.1111/odi.13220

Zucoloto, M. L., Shibakura, M., Pavanin, J. V., Garcia, F. T., da Silva Santos, P. S., Maciel, A. P., de Barros Gallo, C., Souza, N. V., Innocentini, L., Humberto, J., \& Motta, A. (2019). Severity of oral lichen planus and oral lichenoid lesions is associated with anxiety. Clinical Oral Investigations, 23(12), 4441-4448. https://doi.org/10.1007/s0078 4-019-02892-2

\section{SUPPORTING INFORMATION}

Additional supporting information may be found online in the Supporting Information section.

How to cite this article: Adamo D, Calabria E, Coppola N, et al. Psychological profile and unexpected pain in oral lichen planus: A case-control multicenter SIPMO study. Oral Dis. 2021;00:1-17. https://doi.org/10.1111/odi.13787 